To appear in the Journal of Plasma Physics

\title{
Absolute Equilibrium Entropy
}

\author{
By JOHN V. SHEBALIN ${ }^{1}$
}

National Aeronautics and Space Administration, Langley Research Center, Hampton, Virginia 23681, USA

The entropy associated with absolute equilibrium ensemble theories of ideal, homogeneous, fluid and magnetofluid turbulence is discussed and the three-dimensional fluid case is examined in detail. A $\sigma$-function is defined, whose minimum value with respect to global parameters is the entropy. A comparison is made between the use of global functions $\sigma$ and phase functions $H$ (associated with the development of various $H$-theorems of ideal turbulence). It is shown that the two approaches are complimentary though conceptually different: $H$-theorems show that an isolated system tends to equilibrium while $\sigma$-functions allow the demonstration that entropy never decreases when two previously isolated systems are combined. This provides a more complete picture of entropy in the statistical mechanics of ideal fluids.

\footnotetext{
${ }^{1}$ Senior Research Scientist, Aerodynamic \& Acoustic Methods Branch, Mail Stop 128.
} 


\section{Introduction}

The concept of entropy has long been an important and yet a perplexing one. It is important because it never decreases when two previously isolated systems are brought together and hence provides an explicit measure of irreversibility. It is perplexing because although it has long been recognized that "in particular, we cannot speak of its instantaneous value" (Landau \& Lifshitz 1980), this is often done (e.g., Lifshitz \& Pitaevskii 1981). In fact, so-called $H$-theorems explicitly require the existence of a time-dependent function which is assigned the role of a 'dynamic entropy' for an isolated system.

A time-dependent entropy, however, appears to be an oxymoron: entropy measures a certain property of an isolated or quasi-isolated system; the entropy is a constant and does not depend on what specific microstate the system happen to be in at any particular instant. The classical definition of entropy is $S=k_{B} \ln W$, where $k_{B}$ is Boltzmann's constant and $W$ is the number of states available to the system in question. If the system is 'isolated', $W$ is a fixed number; it is immaterial in which of the available states the system resides: the entropy $S$ has a fixed, time-independent value. Of course, the isolated system may interact with other systems and when two or more systems combine into a new system, there is a corresponding, fixed value of entropy for this new system.

Thus, there are two seemingly different views: first, that the entropy of a system is a quantity which is fixed when that system is isolated, and second, that the system, when isolated, has an entropy which evolves from some intial value towards a different 'equilibrium' value. The resolution of this dilemma is to realize that what is evolving is not the entropy itself, but the distribution function $f$ of the system. Concurrently, an estimate of the entropy called an $H$-function $\left[H(f)=-\int f \ln f d \Gamma\right.$ where $d \Gamma$ is an element of the system's phase space] evolves as $f$ does (the evolution of $f$ being determined by a Boltzmann equation). As $f \rightarrow \bar{f}$, where $\bar{f}$ is the equilibrium (or most probable or average) distribution, then $H(f) \rightarrow H(\bar{f})=S$, where $S$ is

the entropy of the system. Showing that $d H(f) / d t \geq 0$ is an important statistical result since it indicates that a system, once isolated, tends to evolve to an equilibrium distribution. Notice that the entropy $S$ does not evolve; rather $H(f)<S$ if $f \neq \bar{f}$ and $H(f) \rightarrow S$ as $f \rightarrow \bar{f}$. In fact, the $H$-function will fluctuate below $S$ as $f$ fluctuates about $\bar{f}$.

The $H$-function is a phase function, that is, a function whose estimate is evolving as the system point moves along a phase trajectory, and entropy is the extremum value of $H$. As Khinchin (1949) emphasizes, 
entropy can also be expressed as the extremum value of a thermodynamic function, i.e., a function of global thermodynamic parameters; here, we will call this function $\sigma$. However, whereas $H$ is a function of the evolving distribution $f$ (a function of time), $\sigma$ is a function of the a priori probability density $D$ (a function of global quantities such as the temperature $T$ ). The difference between the two functions is that while entropy is the maximum value of $H(f)$ with respect to time, it is the minimum value of $\sigma(D)$ with respect to global thermodynamic parameters. This is a very important property of $\sigma$ as it allows the demonstration that entropy never decreases when two isolated systems are combined into one. [Note that this is conceptually different from $d H(f) / d t \geq 0$, which only shows that an isolated system evolves towards equilibrium.]

\section{Statistics of Ideal Fluids}

In this paper, we will be concerned with entropy as related to finite (Fourier) representations of incompressible, homogeneous, ideal (i.e., non-dissipative) fluids and magneto-fluids (so that whenever the words fluid and magneto-fluid are used, incompressibility, homogeneity, and ideality, if not explicitly stated, are always implicit). To illustrate the general concepts, the particular case of an ideal three-dimensional (3-D) fluid will be considered in detail. The goal is to more completely understand how turbulence can be described in terms of statistical mechanics; integral to this understanding is a description of entropy and the role it plays.

It is well known that the classical thermodynamic entropy of each fluid element in an isolated, ideal fluid is conserved (Landau \& Lifshitz 1987); here, we are interested in developing the properties of the absolute equilibrium entropy. The classical entropy, in terms of the probabilities $p_{n}$ that a physical system is in a microstate $n$, is defined as $S=-\sum_{n} p_{n} \ln p_{n}$ (dimensionless units will be used here, so that $k_{B}=1$ ). Similarly, in an absolute equilibrium ensemble, the entropy is determined by finding the extremum of the function $\sigma=-\int D \ln D d \Gamma$, where $D$ is the a priori probability density in the phase space $\Gamma$ whose axes correspond to the independent real and imaginary components of the Fourier velocity coefficients (Shebalin 1989). The probability density $D$ depends on the integral invariants of the motion: for 2-D fluids, these are the energy and enstrophy (Kraichnan 1975, where absolute equilibrium is also discussed); for 2-D magnetofluids, the energy, cross helicity (Woltjer 1958), and mean-squared magnetic potential (Fyfe \& Montgomery 1976, where absolute equilibrium is also discussed); for 3-D fluids, the energy and kinetic helicity (Betchov 1961, Moffatt 1969); and for 3-D magneto-fluids, the energy, cross helicity (Woltjer 1958), and magnetic 
helicity (Elsässer 1956). Additionally, if the mean magnetic field is non-zero, then the mean-squared magnetic potential is not conserved in 2-D ideal magneto-fluids (Shebalin, Matthaeus \& Montgomery 1983), while in 3-D ideal magneto-fluids, the magnetic helicity is no longer an invariant (Shebalin 1994). For 3-D fluids, a detailed discussion of absolute equilibrium was given first by given by Kraichnan (1973), and for 3-D magneto-fluids, a detailed discussion was given first by Frisch, Pouquet, Leorat \& Mazure (1975).

In order to demonstrate that equilibrium (i.e., a most probable state) is attained by an isolated turbulent fluid, several paths have been taken to develop an $H$-theorem. Montgomery used a $B B G K Y$ format to arrive at an H-theorem which shows that a function (here denoted as) $H=-\int f \ln f d \Gamma$ never decreases with time (Montgomery 1976); again, $f$ is the time-dependent distribution function in phase space. An $H$-function for fluid mechanics was also developed by Carnevale, Frisch \& Salmon (1981) and Carnevale (1982). They identify $H_{G}=\frac{1}{2} \sum_{\mathbf{k}} \ln |\mathbf{u}(\mathbf{k})|^{2}$ as an 'entropy functional' [where the $G$ is for $G i b b s$ and the $\mathbf{u}(\mathbf{k})$ are the coefficients associated with a truncated Fourier expansion of the turbulent velocity field]. Again, it is better to call $H_{G}$ an $H$-function rather than entropy function(al) for the reasons given above. A comparison of $H_{G}$ and the $\sigma$-function to be developed will be made and discussed presently.

\section{Absolute Equilibrium}

The equation which describes ideal, incompressible 3 -D fluid dynamics is the Euler equation; in "vorticity form' it is:

$$
\frac{\partial \boldsymbol{\omega}}{\partial t}=\nabla \times(\mathbf{u} \times \boldsymbol{\omega})
$$

Here, the fluid velocity is $\mathbf{u}$ and the vorticity is $\boldsymbol{\omega}=\nabla \times \mathbf{u}$. If the physical variables $\mathbf{u}$ and $\boldsymbol{\omega}$ are expanded in a finite Fourier series, we have, for example:

$$
\mathbf{u}(\mathbf{x})=\sum_{\mathbf{k}} \mathbf{u}(\mathbf{k}) e^{i \mathbf{k} \cdot \mathbf{x}} .
$$

Time dependence of both $\mathbf{u}(\mathbf{x})$ and $\mathbf{u}(\mathbf{k})$ is implicit; also, $\boldsymbol{\omega}(\mathbf{k})=i \mathbf{k} \times \mathbf{u}(\mathbf{k})$ and $\mathbf{u}(\mathbf{k})=i k^{-2} \mathbf{k} \times \boldsymbol{\omega}(\mathbf{k})$. Furthermore, since the velocity field is real, its coefficients must satisfy $\mathbf{u}(\mathbf{k})=\mathbf{u}^{*}(-\mathbf{k})$, where '*' denotes complex conjugation.

Conservation of integral invariants will occur even if the sum in (2) is taken over an arbitrary collection of wave numbers $\mathbf{k}$ (Kraichnan \& Montgomery 1980). The finite set of $\mathbf{k}$ can be chosen so that all $\mathbf{k}$ such 
that $0<|\mathbf{k}| \leq k_{\max }<N / 2$ are included, where $N$ is the order of the Fourier transform in each dimension. This is an isotropic truncation, but it is only one possibility out of many. In particular, two disjoint sets can be created: $\mathbf{k} \in K$ and $\mathbf{k}^{\prime} \in K^{\prime}$, such that $K \neq \emptyset$ and $K^{\prime} \neq \emptyset$ but $K \cap K^{\prime}=\emptyset$; it will, however, be assumed that if $\mathbf{k} \in K$ then $-\mathbf{k} \in K$, and similarly for $K^{\prime}$. Furthermore, let $M$ be the total number of wave vectors in $K$; since $\mathbf{u}(-\mathbf{k})=\mathbf{u}^{*}(\mathbf{k})$, the number of independent wave vectors is $Z=\frac{1}{2} M$.

It was pointed out by T. D. Lee (1952) that the structure of (1), with a periodic solution of the form of (2), admits a statistical solution because a 'Liouville theorem' is satisfied. Again, the components of the independent real and imaginary parts of the coefficients $\mathbf{u}(\mathbf{k})$ are used to label the axes of a multidimensional phase space; the corresponding dynamical system is described by a single point in this phase space, a point which moves about as the system evolves in time. The probability that the system point is in any part of phase space can be described by a canonical probability density $D$ which depends only on a small set of conserved quantities, the integral invariants of the dynamical system. Once $D$ has been found, then the equilibrium energy spectrum can be determined, even though the $\mathbf{u}(\mathbf{k})$ are random variables.

In the case of isotropic, incompressible 3 -D Euler turbulence, the integral invariants are the energy $E$ and the kinetic helicity $H_{k}$ (Betchov 1961):

$$
E=\frac{1}{2} \sum_{\mathbf{k} \in K}|\mathbf{u}(\mathbf{k})|^{2} \quad \text { and } \quad H_{k}=\frac{1}{2} \sum_{\mathbf{k} \in K} \mathbf{u}(\mathbf{k}) \cdot \boldsymbol{\omega}^{*}(\mathbf{k})
$$

while the absolute equilibrium probability density (Kraichnan 1973) is

$$
D=C \exp \left(-\alpha E-\beta H_{k}\right) \quad \text { where } \quad C=\prod_{\mathbf{k} \in K_{Z}}\left(\frac{\alpha^{2}-\beta^{2} k^{2}}{\pi^{2} k^{4}}\right)^{\frac{3}{2}} .
$$

The product above is taken over the set $K_{Z}: \mathbf{k} \in K_{Z}$ implies $\mathbf{k} \in K$, but $-\mathbf{k} \notin K_{Z}$; thus, $K_{Z}$ has half the elements of $K$. (To create $K_{Z}$, start with $K_{Z}$ empty and then choose each $\mathbf{k} \in K$ in turn and place it into $K_{Z}$, if $\mathbf{- k}$ is not already there.)

The model system, in which ideal turbulence is simulated numerically by integrating a finite Fourier representation of equation (1) forward in time, is canonical as it can be considered a small system (a computer code) weakly interacting with a larger 'heat bath' (a digital computer). The 'weak interaction' is due to numerical round-off and time-discretization errors, which cause very small fluctuations of $E$ and $H_{k}$; it is within this context that these are called 'integral invariants' of a 'quasi-closed' system. 
Using the probability density $D$ in (4), the expectation value of any quantity $Q$ can be defined as

$$
\langle Q\rangle \equiv \int Q D d \Gamma, \quad \text { where } \quad d \Gamma=\prod_{\mathbf{k} \in K_{Z}} d \mathbf{u}_{R}(\mathbf{k}) d \mathbf{u}_{I}(\mathbf{k})
$$

where the integration limits on each dimension are $-\infty$ to $\infty$, and where the subscripts $R$ and $I$ denote real and imaginary parts, respectively, of the complex coefficients. Using (5), expectation values of the moments of the Fourier coefficients can be easily found (since $D$ is essentially gaussian):

$$
\begin{gathered}
\left\langle\mathbf{u}_{R}(\mathbf{k})\right\rangle=\left\langle\mathbf{u}_{I}(\mathbf{k})\right\rangle=0, \quad\left\langle\left|\mathbf{u}_{R}(\mathbf{k})\right|^{2}\right\rangle=\left\langle\left|\mathbf{u}_{I}(\mathbf{k})\right|^{2}\right\rangle=\frac{3 \alpha}{2\left(\alpha^{2}-\beta^{2} k^{2}\right)}, \\
\text { and } \quad\left\langle\mathbf{u}_{R}(\mathbf{k}) \cdot \boldsymbol{\omega}_{R}(\mathbf{k})\right\rangle=\left\langle\mathbf{u}_{I}(\mathbf{k}) \cdot \boldsymbol{\omega}_{I}(\mathbf{k})\right\rangle=\frac{-3 \beta k^{2}}{2\left(\alpha^{2}-\beta^{2} k^{2}\right)},
\end{gathered}
$$

where all the components of $\mathbf{u}(\mathbf{k})$ for a given $\mathbf{k}$ have equal expectation values. Substituting (6) into (3) gives the expectation values

$$
\langle E\rangle=\frac{3}{2} \sum_{\mathbf{k} \in K} \frac{\alpha}{\alpha^{2}-\beta^{2} k^{2}} \quad \text { and } \quad\left\langle H_{k}\right\rangle=-\frac{3}{2} \sum_{\mathbf{k} \in K} \frac{\beta k^{2}}{\alpha^{2}-\beta^{2} k^{2}}
$$

Since the energy and kinetic helicity are canonical invariants, they are conserved to within very small fluctuations, and their values over time are essentially the same as either their initial or time-averaged values. Thus, the relations (7) implicitly determine the 'inverse temperatures' $\alpha$ and $\beta$ in terms of $\langle E\rangle$ and $\left\langle H_{k}\right\rangle$.

Other global quantities, such as the enstrophy $\Omega$, will generally have large fluctuations; nevertheless, the enstrophy also has an expectation value:

$$
\Omega=\frac{1}{2} \sum_{\mathbf{k} \in K} k^{2}|\mathbf{u}(\mathbf{k})|^{2} \Rightarrow\langle\Omega\rangle=\frac{3}{2} \sum_{\mathbf{k} \in K} \frac{\alpha k^{2}}{\alpha^{2}-\beta^{2} k^{2}} .
$$

Using (7) and (8), the following algebraic relations are arrived at:

$$
\alpha\langle E\rangle+\beta\left\langle H_{k}\right\rangle=\frac{3}{2} \sum_{\mathbf{k} \in K} 1=3 Z, \quad \alpha\left\langle H_{k}\right\rangle+\beta\langle\Omega\rangle=0
$$

Thus, both $\alpha$ and $\beta$ can be considered functions of one quantity, $\langle\Omega\rangle$ :

$$
\alpha=\frac{3 Z\langle\Omega\rangle}{\langle E\rangle\langle\Omega\rangle-\left\langle H_{k}\right\rangle^{2}} \quad \text { and } \quad \beta=\frac{-3 Z\left\langle H_{k}\right\rangle}{\langle E\rangle\langle\Omega\rangle-\left\langle H_{k}\right\rangle^{2}}
$$

Using (3) and (8), it is straightforward but tedious to show that $\langle E\rangle\langle\Omega\rangle-\left\langle H_{k}\right\rangle^{2} \geq 0$; thus, from (10), $\alpha>0$ and the product $\beta\left\langle H_{k}\right\rangle \leq 0$. Furthermore, under coordinate inversion, $\left\langle H_{k}\right\rangle \rightarrow-\left\langle H_{k}\right\rangle$, while neither $\langle E\rangle$ nor 
$\langle\Omega\rangle$ change sign; thus, though $\alpha$ is a scalar, $\beta$ is a pseudoscalar under a parity transformation (i.e., coordinate inversion). Note that (1) is invariant under a parity transformation; the fact that $\beta$ is a pseudoscalar ensures that the probability distribution (4) is also invariant. Also, for ideal magneto-fluids, the ratio $R$ of magnetic energy to kinetic energy is used in place of $\Omega$ (Shebalin 1989, 1994; Stribling \& Matthaeus 1990).

\section{Entropy}

At this point we are in a position to define the entropy of an absolute equilibrium ensemble and to develop its properties. Consider now the expectation value of $\ln D$; using (4), (5), and (9),

$$
\begin{aligned}
\sigma=-\langle\ln D\rangle & =-\int D \ln D d \Gamma \\
& =\alpha\langle E\rangle+\beta\left\langle H_{k}\right\rangle-\ln C \\
& =3 Z-\ln C .
\end{aligned}
$$

Note that $\sigma$ is a function of $\alpha$ and $\beta$ through (4). In turn, $\alpha$ and $\beta$ are determined by (10); however, in examining (10), it is clear that $\alpha$ and $\beta$ cannot be determined unless $\langle E\rangle,\left\langle H_{k}\right\rangle$, and $\langle\Omega\rangle$ are known. Prior to performing a numerical simulation (Shebalin 1989,1994), however, all that is known is that $\langle E\rangle$ and $\left\langle H_{k}\right\rangle$ are constants, while $\langle\Omega\rangle$ is unknown. In this case, set $\langle E\rangle=\bar{E},\left\langle H_{k}\right\rangle=\bar{H}_{k}$, and $\langle\Omega\rangle=\Omega$; then $\bar{E}$ and $\bar{H}_{k}$ can be taken as the initial values (which are known when the initial conditions are known), and the value of $\Omega$ is variable, with $\bar{H}_{k}^{2}<\Omega \leq k_{\text {max }}^{2} \bar{E}$.

Using the above assignments, the inverse temperatures $\alpha$ and $\beta$, as given in (10), become:

$$
\alpha=\frac{3 Z \Omega}{\bar{E} \Omega-\bar{H}_{k}^{2}} \quad \text { and } \quad \beta=\frac{-3 Z \bar{H}_{k}}{\bar{E} \Omega-\bar{H}_{k}^{2}} .
$$

Also, using (4) and (11), the dependence of $\sigma$ on these inverse temperatures takes the form:

$$
\sigma=\sigma_{o}-\frac{3}{2} \sum_{\mathbf{k} \in K_{Z}} \ln \left(\alpha^{2}-\beta^{2} k^{2}\right) \quad \text { where } \sigma_{o}=3 Z(1+\ln \pi)+3 \sum_{\mathbf{k} \in K_{Z}} \ln k^{2} .
$$

Putting (12) into (13), $\sigma$ is seen to be explicitly a function of only one variable parameter, $\Omega: \sigma=\sigma(\Omega)$.

Although we now have $\sigma(\Omega)$, what is the advantage in this? In other words, why not solve the system of equations (7) directly for $\alpha$ and $\beta$, place these into $C$ as given by (4), and put this, in turn, into (11), giving the entropy $S=\sigma$ ? The advantage, as Khinchin (1949) points out, is that we can now demonstrate that entropy never decreases when two previously isolated systems are brought together. 
The function $\sigma=3 Z-\ln C$ defined in (11), and more explicitly in (13), is exactly analogous to (83) of Khinchin (1949). It can be shown directly, or by referring to the more general results of Khinchin (1949, pp. 76-77), that $\sigma(\Omega)$ has only one extremum $\sigma^{\prime}(\Omega)=d \sigma / d \Omega=0$ at $\Omega=\bar{\Omega}$ and that $\sigma^{\prime \prime}(\bar{\Omega})>0$. Thus, $\Omega=\bar{\Omega}$ is a point of global minimum for $\sigma(\Omega)$; the entropy of the canonical system is $S=\sigma(\bar{\Omega})$. All of this allows a direct proof that entropy increases when two isolated systems are brought together (Khinchin 1949).

Consider two sets of wave vectors $K^{(1)}$ and $K^{(2)}$ such that $K^{(1)} \cap K^{(2)}=\emptyset$ and $K^{(1)} \cup K^{(2)}=K$; also, $M=M^{(1)}+M^{(2)}$ and $Z=Z^{(1)}+Z^{(2)}$. Looking at $(3),(4),(5)$, and (8), it is clear that

$$
\begin{gathered}
E=E^{(1)}+E^{(2)}, \quad H_{k}=H_{k}^{(1)}+H_{k}^{(2)}, \quad \Omega=\Omega^{(1)}+\Omega^{(2)}, \\
D=D^{(1)} D^{(2)}, \quad C=C^{(1)} C^{(2)}, \quad d \Gamma=d \Gamma^{(1)} d \Gamma^{(2)} .
\end{gathered}
$$

Using these results, along with (11) gives us

$$
\sigma^{(1)}+\sigma^{(2)}=3\left[Z^{(1)}+Z^{(2)}\right]-\ln \left[C^{(1)} C^{(2)}\right]=Z-\ln C=\sigma
$$

ı.e., $\sigma$ is an additive function.

In terms of explicit arguments, we have

$$
\sigma(\Omega)=\sigma^{(1)}\left(\Omega^{(1)}\right)+\sigma^{(2)}\left(\Omega^{(2)}\right)
$$

After the subsystems have been combined, a minimum for the function $\sigma(\Omega)$ will occur at some value $\Omega=\bar{\Omega}$; since we keep track of all the modes of the system, $\bar{\Omega}$ can be uniquely written as a sum of two parts: $\bar{\Omega}=\bar{\Omega}^{(1)}+\bar{\Omega}^{(2)}$, where $\bar{\Omega}^{(1)}$ and $\bar{\Omega}^{(2)}$ correspond to subsystems 1 and 2 , respectively. Before the subsystems were combined, however, they had individual minima at $\bar{\Omega}_{0}^{(1)}$ and $\bar{\Omega}_{o}^{(2)}$; therefore,

$$
\sigma^{(1)}\left(\bar{\Omega}^{(1)}\right) \geq \sigma^{(1)}\left(\bar{\Omega}_{o}^{(1)}\right) \quad \text { and } \quad \sigma^{(2)}\left(\bar{\Omega}^{(2)}\right) \geq \sigma^{(2)}\left(\bar{\Omega}_{o}^{(2)}\right)
$$

From this it immediately follows that the total entropy after combination is greater than or equal to the sum of the individual entropies $S_{0}^{(1)}$ and $S_{0}^{(2)}$ which existed before combination:

$$
\begin{aligned}
S=\sigma(\bar{\Omega}) & =\sigma^{(1)}\left(\bar{\Omega}^{(1)}\right)+\sigma^{(2)}\left(\bar{\Omega}^{(2)}\right) \\
& \geq \sigma^{(1)}\left(\bar{\Omega}_{0}^{(1)}\right)+\sigma^{(2)}\left(\bar{\Omega}_{0}^{(2)}\right)=S_{0}^{(1)}+S_{o}^{(2)}
\end{aligned}
$$

Thus, we have shown, for ideal fluid turbulence, that the quantity that has been defined as the entropy of an absolute equilibrium ensemble satisfies the necessary condition that the total entropy is always greater 
than or equal to the sum of the entropies of the two systems prior to their interaction: $S \geq S_{0}^{(1)}+S_{0}^{(2)}$. Note that there is no 'time evolution' occurring here: the instant two previously isolated systems are merged, the entropy of the new combined system is established. The new system may then evolve from one microstate to another 'more probable' one; the new entropy, however, remains fixed in value.

\section{Discussion}

An analogous discussion of $H(f)=-\int f \ln f d X$ can be given: $H$ can take different values, depending on the instantaneous form of $f$; however, only one particular value of $f$ corresponds to an equilibrium, or average, value. Let this value be denoted by $\bar{f}$ : the entropy is $S=H(\bar{f})$. If the system is started off at some less probable distribution, denoted by $f_{0}$, then $H$ has the value $H\left(f_{0}\right)$, which is not the entropy, since an isolated system (in a canonical sense) has only one value for its entropy, $H(\bar{f})$.

Both $\sigma$ and $H$ can be called 'pre-entropies' as it is imprecise to call them 'entropies.' The function $H(f)$ increases as $f \rightarrow \bar{f}$ until it reaches a maximum of $H(\bar{f})$ : this is the entropy. The function $\sigma(\Omega)$ decreases as $\Omega \rightarrow \bar{\Omega}$ until it reaches a minimum of $\sigma(\bar{\Omega})$ : this is also the entropy. Both $H(f)$ and $\sigma(\Omega)$ may fluctuate since the instantaneous form of $f$ and the instantaneous value of $\Omega$ fluctuate. Neither of these, however, are the entropy for an isolated system, which has a fixed, non-fluctuating value.

The function $\sigma(\Omega)$ is useful, as Khinchin (1949) points out, and as was shown in (18), in that it can be used to demonstrate the non-decreasing nature of entropy. It is also useful because the inverse of the normalizing coefficient $C$, as given in (4), is the so-called partition function of statistical mechanics (Landau \& Lifshitz 1980, p. 91), and thus, connects the statistical analysis of ideal fluids to the main body of classical statistical mechanics. Furthermore, if we use (11) to write $S=\alpha \bar{E}+\beta \bar{H}_{k}-\ln C(\bar{\Omega})$, then we have $\partial S / \partial \bar{E}=\alpha$ and $\partial S / \partial \bar{H}_{k}=\beta$, which generalizes the well-known thermodynamic result $d S / d E=1 / T$ (Landau \& Lifshitz 1980, p. 35) to the case of more than one temperature (here, $T \rightarrow \alpha^{-1}, \beta^{-1}$ ).

Finally, a comment on the entropy function $H_{G}=\frac{1}{2} \sum_{\mathbf{k}} \ln |\mathbf{u}(\mathbf{k})|^{2}$ of Carnevale, Frisch \& Salmon (1981) and Carnevale (1982). On looking at (6), we see that $|\mathbf{u}(\mathbf{k})|^{2}=3 \alpha /\left(\alpha^{2}-\beta^{2} k^{2}\right)$; then, from (13), $H_{G}-$ $2 \sigma / 3 \sim Z \ln \alpha$. Thus, the entropy function $H_{G}$ appears to have an additional term when compared with $\sigma$, and is therefore inconsistent with absolute equilibrium ensemble theory. However, $\beta \rightarrow 0$ in the limit of zero kinetic helicity, and the essential difference between $H_{G}$ and $\sigma$ disappears. 


\section{Conclusion}

In this paper, we have developed the notion of entropy within the framework of the absolute equilibrium ensemble theory of ideal fluids. This has enabled a discussion of the distinction between the entropy $S$ of an isolated system and the quantities $H$ and $\sigma: S$ is independent of time for an isolated system, while $H$ is a time-dependent measure of a system's evolution to a 'most probable' state, and $\sigma$ allows for the analytical demonstration of the law of increase of entropy when two isolated systems are combined. Recognizing the distinction between $H$ and $\sigma$ also obviates Loschmidt's paradox, which is discussed by Carnevale (1982).

Although much reference was given here to the work of Khinchin (1949), it should be noted that there are more modern treatments of this subject (e.g., Sinai 1994). However, these modern works have a much more abstruse character for non-mathematicians; this, along with the general completeness, for our purposes, of the work of Khinchin (1949), has guided our choice of reference. The book by Khinchin (1949), translated by Gamow, is well worth reading. 


\section{REFERENCES}

Betchov, R. 1961 Phys. Fluids 4, 925.

Carnevale, G. F., Frisch, U. \& Salmon, R. 1981 J. Phys. A 14, 1701.

Carnevale, G. F. 1982 J. Fluid Mech. 122, 143.

Elsässer, W. M. 1956 Rev. Mod. Phys. 28, 135.

Frisch, U., Pouquet, A., Leorat, J. \& Mazure, A. 1975 J. Fluid Mech. 68, 769.

Fyfe, D. \& Montgomery, D. 1976 J. Plasmas Phys. 16, 181.

Khinchin, A. I. 1949 Mathematical Foundations of Statistical Mechanics, pp. 137-145, Dover, New York.

Kraichnan, R. H. 1973 J. Fluid Mech. 59, 745.

Kraichnan, R. H. 1975 J. Fluid Mech. 67, 155.

Kraichnan, R. H. \& Montgomery, D. 1980 Rep. Prog. Phys. 43, 547.

Landau, L. D. \& Lifshitz, E. M. 1980 Statistical Physics, p. 27, Pergamon, New York.

Landau, L. D. \& Lifshitz, E. M. 1987 Fluid Mechanics, pp. 3-4, Pergamon, New York.

LeE, T. D. 1952 Q. Appl. Math. 10,69.

Lifshitz, E. M. \& Pitaevskit, L. P. 1981 Physical Kinetics, p. 11, Pergamon, New York.

Moffatt, H. K. 1969 J. Fluid Mech. 35, 117.

Montgomery, D. 1976 Phys. Fluids 19, 802.

Shebalin, J. V., Matthaeus, W. H. \& Montgomery, D. 1983 J. Plasma Phys. 29, 525.

Shebalin, J. V. 1989 Physica D 37, 173.

Shebalin, J. V. 1994 Phys. Plasmas 1, 541.

Sinai, Ya. G. 1994 Topics in Ergodic Theory, Princeton U. P., Princeton, New Jersey.

Stribling T. \& Matthaeus, W. H. 1990 Phys. Fluids B 2, 1979.

WoltJer, L. 1958 Proc. Nat. Acad. Sci. U.S.A. 44, 833. 